\title{
Assessment of two hemispherical and hemispherical-conical miniature sources used in electronic brachytherapy using Monte Carlo Simulation
}

\author{
Barat Barati ${ }^{1}$, Mansour Zabihzadeh ${ }^{2}$, Mohammad Javad Tahmasebi Birgani ${ }^{3}$, Nahid Chegini ${ }^{4}$, Mojtaba Hoseini \\ Ghahfarokhi $^{5}$, Jafar Fatahiasl ${ }^{6}$
}

\footnotetext{
${ }^{1}$ Ph.D. Candidate, Department of Medical Physics, School of Medicine, Ahvaz Jundishapur University of Medical Sciences, Ahvaz, Iran

${ }^{2}$ Ph.D. of Medical Physics, Assistant Professor, Department of Medical Physics, School of Medicine, Ahvaz Jundishapur University of Medical Sciences, Ahvaz, Iran

${ }^{3}$ Ph.D. of Medical Physics, Professor, Department of Medical Physics, School of Medicine, Ahvaz Jundishapur University of Medical Sciences, Ahvaz, Iran

${ }^{4}$ Ph.D. of Medical Physics, Assistant Professor, Department of Medical Physics, School of Medicine, Ahvaz Jundishapur University of Medical Sciences, Ahvaz, Iran

${ }^{5}$ Ph.D. Candidate, Member of Student Research Committee, Department of Medical Physics, School of Medicine, Ahvaz Jundishapur University of Medical Sciences, Ahvaz, Iran

${ }^{6}$ Ph.D. of Medical Physics, Assistant Professor, Department of Para Medicine, School of Medicine, Ahvaz Jundishapur University of Medical Sciences, Ahvaz, Iran
}

\section{Type of article: Original}

\begin{abstract}
Introduction: Since the heart of the electronic brachytherapy system is a tube of a miniature $\mathrm{x}$-ray and due to the increasing use of electronic brachytherapy, there is an urgent need for acquiring knowledge about the X-ray spectrum produced, and distribution of $\mathrm{x}$-ray dose. This study aimed to assess the optimal target thickness (TT), the X-ray source spectrum, and the absorbed dose of two miniature sources of hemispherical and hemisphericalconical used in electronic brachytherapy systems, through a Monte Carlo simulation.

Methods: Considering the advantages of MCNPX Code (2.6.0), two input files corresponding to the characteristics of the investigated miniature sources were prepared for this code and then were used for simulation. The optimal thickness (OT) of gold and tungsten targets was determined for the energy levels of 40, 45, and 50 kilo-electron-volts.

Results: In this study, the values of the size of the optimal thickness of $0.92,1.01$ and $1.06 \mu$ for gold target and values of $0.99,1.08$ and $1.34 \mu$ for tungsten target were obtained for energies 40,45 and $50 \mathrm{keV}$ that using these values, the optimum thickness of 0.92 , X-ray spectrum within and outside targets, axial and radial doses for the used energy were calculated for two miniature sources.

Conclusion: It was found that the energy of incident electron, target shape, cross-sectional area of the produced bremsstrahlung, atomic number of materials constituting of the target and output window are the factors with the greatest impacts on the produced X-ray spectrum and the absorbed dose.
\end{abstract}

Keywords: Mont Carlo simulation; Electronic Branchy therapy; Energy Spectrum; Dose calculation; Mesh tally

\section{Introduction}

The aim of radiation therapy is the application of ionizing radiation to treat or control the growth of cancer cells forming a tumor (1). In comparison to external beam radiation therapy (EBRT), brachytherapy allows the exposure to radiation to be concentrated on certain organs, but this comes at the expense of a more invasive treatment (2). There are problems associated with this method, like the chance of radioactive seed escaping the treatment zone and

\section{Corresponding author:}

Barat Barati, Department of Medical Physics, School of Medicine, Ahvaz Jundishapur University of Medical Sciences, Ahvaz, Iran. Tel: +98.9163014685, Fax:+98.6133332036, Email: barati943@yahoo.com

Received: September 27, 2016, Accepted: December 22, 2016, Published: February 2017

iThenticate screening: December 02, 2016, English editing: February 05, 2017, Quality control: February 12, 2017

(C) 2017 The Authors. This is an open access article under the terms of the Creative Commons Attribution-NonCommercialNoDerivs License, which permits use and distribution in any medium, provided the original work is properly cited, the use is non-commercial and no modifications or adaptations are made. 
affecting the neighboring tissues (3). Another challenge concerning this method is that dose distribution on the area of interest is quite ambiguous (4), and dose rate and depth-dose profile of each radioisotope source are constant (5), also when using radionuclide-based source, there is no way to turn off radiation production whether it is needed or not (6). In the 1980s, collaboration between Alan Sliski and his team at Photoelectron and MIT resulted in the development of an Electronic Brachytherapy System (eBS) (7), which managed to address some of the above problems by using an alternative point source radiation treatment approach (8). The advantages of eBS in comparison to radionuclide-based brachytherapy include: unlike radionuclide-based sources, in eBS radiation is generated by an X-ray tube (9); the therapy dose is adjustable as it can be altered by adjusting the current and voltage of X-ray tube (10); the use of lower energies in eBS means increased radiobiological effectiveness and that surrounding environment can be better protected by less extensive shielding (11); the dose distributions can be controlled more accurately with the tube's $\mathrm{kVp}$ adjustment (12); the use of eBS allows dose distribution to be targeted more accurately and the dose fall-off rate to be better controlled, and this can prevent unnecessary delivery of dose to marginal healthy tissues (13); In eBS, the tube potential is adjustable, which gives better control over radiation quality and leads to a better dose conformity (2). The Zeiss INTRABEAM ${ }^{\circledR}$ Radiotherapy System (IRS) is a $50 \mathrm{kV}$ X-ray unit designed specifically for interstitial brachytherapy (14). However, considering the problems associated with the use of brachytherapy isotopes, including iridium-192 used for treatment of breast cancer via MammoSite method, in 2005, Xoft Company introduced the miniature x-ray tube as a response to these issues. Axxent ${ }^{\mathrm{TM}}$ Electronic Brachytherapy System delivers focused, conformal doses of radiation at high dose rates using a novel miniature $\mathrm{x}$-ray source operating at 40,45 or $50 \mathrm{kVp}(15)$. Characteristics of this system make it particularly useful for treatment of cancer developed in the breast and the inner surface of a body cavity like the bed of an excised tumor (16). In 2013, the Esteya brachytherapy system was introduced for the treatment of skin. All three, the Zeiss Intrabeam system (Carl Zeiss, Oberkochen, Germany), the Axxent system (Xoft, Fremont, CA) and the Esteya Electronic Brachytherapy System (Elekta AB-Nucletron, Stockholm, Sweden) mentioned above, use particular photon emitting sources, for which Task Group-186 of the American Association of Physicists in Medicine (AAPM) have developed a number of guidelines (17). The AAPM has also provided exact definitions for the Miniature Electronic Brachytherapy X-ray Sources (MEBXS) emitting a spectrum of bremsstrahlung and characteristic X-ray photons (18). The work of Task Group 152 of AAPM has resulted in a model regulation for electronic brachytherapy, where the term "electronic brachytherapy" has been defined as "a method of radiation therapy using electrically generated $\mathrm{x}$-rays to deliver a radiation dose at a distance of up to a few centimeters by intracavitary, intraluminal, or interstitial application, or by applications with the source in contact with the body surface or very close to the body surface" (19). Through these developments, electronic brachytherapy system has emerged as one of the most rapidly disseminating medical devices in the world. However, the core component of these systems is still the miniature tube that allows the low energy $\mathrm{x}$-ray to be produced at high dose rates (20). The X-ray spectrum produced by this tube has a continuous distribution (bremsstrahlung effect) with a line spectrum characteristic (21), and has an extensive use in medical physics (22). There are several concerns regarding the electronic brachytherapy, and one of these concerns is the manner in which the X-ray dose is distributed. This issues a major concern because for the treatment to be efficient, the dose should be delivered uniformly into the cavity or area of interest. As a result, the geometry and the structure of the target used in the X-ray tube of eBS plays a central role in the delivered dose distribution (9). Thus, the aim of the study was to compare the optimal target thickness, the energy spectrum at different positions, presence of beryllium window, and the relative dose of hemispherical and hemispherical-conical sources using the functions of MCNPX code (2.6.0).

\section{Material and Methods}

\subsection{Simulation of miniature $X$-ray sources}

The utility of Monte Carlo (MC) simulation for the study of physics of nuclear medicine, radiology, and radiation therapy is a dominantly accepted fact (23). The ability of Monte Carlo codes to model the full geometric detail of real world systems has also been proven (24). The transport of neutron, photon and electron though a threedimensional medium with arbitrary material and geometry, can be simulated with a general-purpose Monte Carlo NParticle code (25). One tool for such simulation is the MCNPX code, which is a combination of MCNP4C code and the code developed by LAHET (Los Alamos High-Energy Transport) for the transport and interaction of nucleons, pions, muons and light ions in complex geometries. All desirable features of MCNP4C and MCNP5 have been incorporated into the MCNPX code, but in addition, this code covers the transport of 34 new types of particles (protons, light ions, etc.), and so expands the particle-energy range that can be simulated (26). This general-purpose radiation transport code can cover continuous-energy transport up to one $\mathrm{TeV}$, and provides several sources and tally options. In addition, MCNPX utilize interactive graphics, and can be run with both sequential and parallel processors (27). The version 2.6 of MCNPX requires even less memory than the previous versions, and this allows it 
to simulate larger voxel phantoms. However, voxel-based phantoms of large matrix sizes still cannot be simulated by MCNP5 (28). In this study, the code MCNPX (version 2.6.0) was used to simulate the target thickness, the generated x-ray photon spectra and the dose delivered through 2 targets of different material. A file containing cell card, surface card, and data card was written based on the geometry of hemispherical and hemispherical-conical sources, and was then used for simulation with MCNPX (2.6.0). Simulations were based on cylinders with internal radius (the target), middle radius (the output window), and outer radius (the water cooling sheath) of 1.1, 1.6, and $2.7 \mathrm{~mm}$, respectively. Densities of gold, tungsten, beryllium, and water were assumed to be 19.32, 19.3, 1.848, $0.998, \mathrm{~g} / \mathrm{cm}^{3}$ respectively (29). Considering the necessity of bremsstrahlung production for extracting an output, all stages of coupled electron/photon transport was simulated with the MODE: P E card. This study employed the energy cut-off approach, which is a simple variance reduction method, where any particle whose energy is lower than a threshold will be disregarded. Simulating these cut-off particles can give more (yet unnecessary) information, but eliminating them can improve computation time without significant consequences (28). In all stages of simulations, the cut-off energy was assumed to be $1 \mathrm{keV}$ for both electrons and photons. In the simulations, the length of the source $(15 \mathrm{~mm})$ was modeled along the z-axis with its bottom (start) coinciding with the origin. The source was considered to be symmetric about the z-axis to facilitate the calculation dose delivery with the MC code. The reason behind the selection of this configuration was the presence of ITS-style in MCNP. ITS-style employs the transport parameters that belong to the energy step of the nearest interruption, and leads to better results than the MCNP-style (30). As a result, DBCN $17 \mathrm{j} 1$ Card was defined accordingly in the input file. Library Cards Plib $=04 \mathrm{P}$ and elib $=03 \mathrm{e}$ were also used in the input file in accordance with the MCNPX (2.6.0) manual. The materials such as tungsten, molybdenum, and rhodium with different atomic numbers and densities, can be good candidates for the Xrays targets. Currently, the Intrabeam system (hemispherical source) uses a gold target $(\mathrm{z}=79)$ because of good heat conductance, but the reason behind choosing tungsten for hemispherical-conical is that it has a high atomic number and a high melting point (3422C) (31), which ensure not only an efficient $\mathrm{x}$-ray production but also a good tolerance for high power deposition (32). It should also be noted that $\mathrm{x}$-ray production efficiency is correlated with the square of the atomic number of source $\left(Z^{2}\right)$, and so the atomic numbers of tungsten $(Z=74)$ and gold $(z=79)$ make them a good choice for this purpose (33). Tungsten also has good strength and ductility and a low rate of vaporization in vacuum (34). The miniature sources simulated with Monte Carlo code MCNPX (2.6.0) and their specific input files are shown in Figure 1.
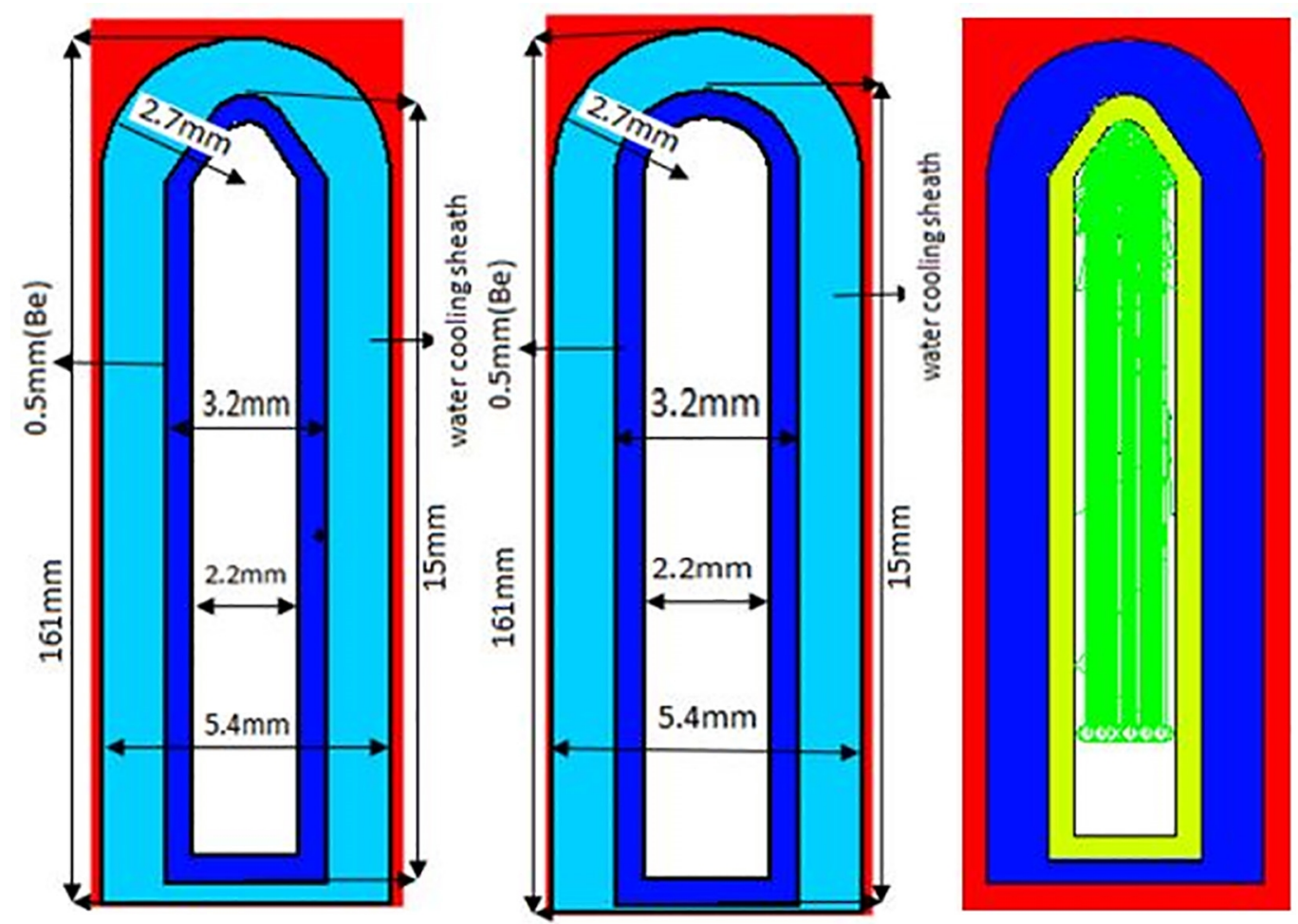

Figure 1. A view of simulated miniature sources; electron beams path (green color) 


\subsection{Computation of optimal thickness of Targets}

In this part of the study, as Figure 2 (A, B, and C) shows, the targets of different thicknesses (without the output window and water cooling sheath) and with sizes specified in the input file, were tested for energy levels of 40, 45 and $50 \mathrm{keV}$ using MCNPX Visual Editor Software with electron beam particle display. The electron penetration (into the thickness of the target) and particle track plots were used to determine a rough range for the desirable thickness, and thus provide a ground for quantitative and more accurate determination of size of OT using the code. The process of determining the optimal angle of the hemispherical-conical source was started by setting up the interface of two cones with two hemispheres. The outer hemisphere had a fixed radius of $0.4 \mathrm{~mm}$ and the other one had a variable radius. The outer cone was cut by a cylinder with a radius of $1.1 \mathrm{~mm}$ and the other one was cut by a thinner cylinder with variable radius. The bottom end of the latter cylinder was cut by the plane that was the measure of distance between the target and the electron source. Once this slightly complex structure was set up, the desired solid disc-shaped electron source was applied using the Monte Carlo code, and the optimal angle of the hemispherical-conical source was determined to be in the range of 33 to 34 degrees depending on the used energy level. Note that any change in any of the described variables alters the apex of the cone, and changes the obtained angle. The optimized angles obtained for the hemispherical-conical and the rough approximations obtained for TT were used along with the 0.8 micron disk source (15), to simulate two sources with 30 thicknesses ranging from 0.1 microns to 3 microns (increasing in 0.1 steps), and with energy levels of 40,45 , and $50 \mathrm{keV}$ (increasing in $5 \mathrm{keV}$ steps) by running 180 instances of 300 minute programs. Programs were run in groups of 6 on a computer with Intel (R) Core ${ }^{\mathrm{TM}}$ I 7-4790k CPU@4.00GHz, and 16.0 GB Ram. For each target, the surface with which particles (namely electrons and photons) were colliding was named the 'input surface' and the surface in which bremsstrahlung was being produced was named the 'output surface'. The tallies F1 and F2 were considered for electrons colliding with the input surface, and the tallies *F1 and *F2 were considered for electrons in the output surface. The distance between electron source and the surface that cuts the lower end of the innermost cylinder was $10 \mathrm{~mm}$. In all runs, the error of the process was less than $2 \%$ and in some tallies it reached down to a hundredth of one percent.
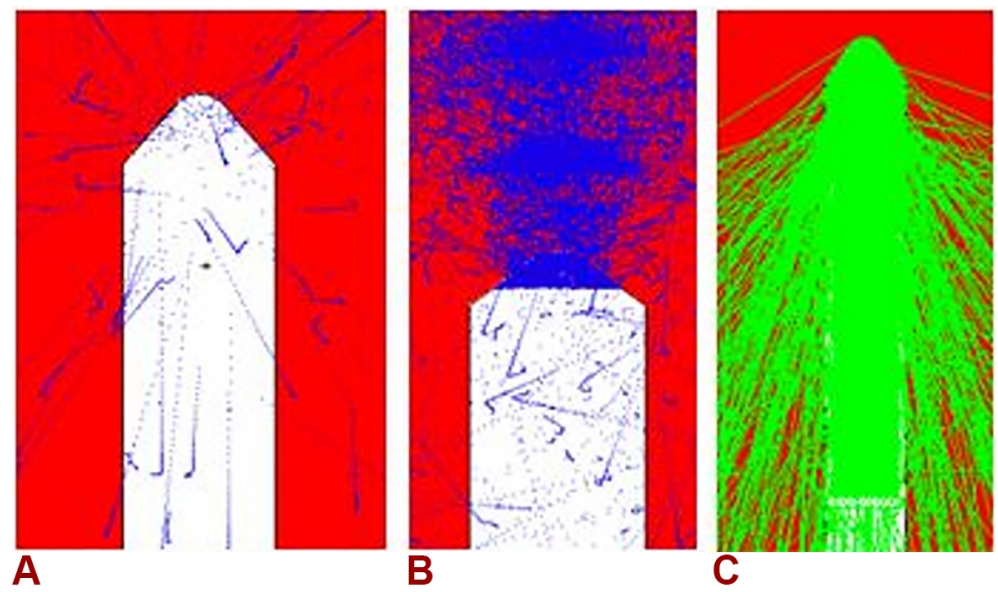

Figure 2. Targets used for qualitative determination of the range of target thickness

\subsection{Computation of spectra resulting from the miniature sources}

In MCNPX, Physical parameters expressed by the cards PHYS: E and PHYS: P can be used to improve the efficiency of electron/photon transport computations. This task is achieved by simplifying the less important physical aspects of the model (35). The codes were run with a default photon - electron physics card. However, Emax was assumed to be $1 \mathrm{keV}$ higher than what is defined in the electron source profile and specified in the data card. For example, when the energy of electron source was $50 \mathrm{keV}$, the Emax of the photon - electron PHYS card was assumed to be $51 \mathrm{keV}$ to avoid calculation errors. The ring detector tally has been defined as a point detector tally where the location of the point detector is variable but can be sampled from some location on the ring. The use of ring detector leads to improved performance of point detector for those problems in which geometrical structures have rotational symmetry with respect to one axis (36). Considering the good features of the ring tally F5 and symmetry of miniature sources about z-axis, this tally was used for the calculation of energy spectra. The other issue that needed to be considered was the material of $0.5 \mathrm{~mm}$ thick output window (substrate). Because of the lack of accurate knowledge about the material of this component in the miniature sources of Xoft Company, this component was assumed to be made of beryllium. This material was selected because of features such as good strength to 
weight ratio, good thermal strength, transparency to shorter wavelengths, good availability (37), low atomic number and its ability to let through even the lower energy X-rays without absorption (33), and also the fact that the Zeiss Company usually uses beryllium as the substrate or heat buffer. To calculate the spectra of sources, one time - just the target alone was used (Figure 2, B), and another time - the target, beryllium window, and water cooling sheath again (Figure 1) were used. This means that spectra were calculated both inside and outside the target and beyond and within the water cooling sheath. Figures 3 and 4 show the obtained spectra. In the course of calculating the spectra, the energy peaks were determined with the help of bin card and with a step of $0.1 \mathrm{keV}$. Also, the miniature sources were assumed to be in a sphere of air with a radius of $20 \mathrm{~cm}$.
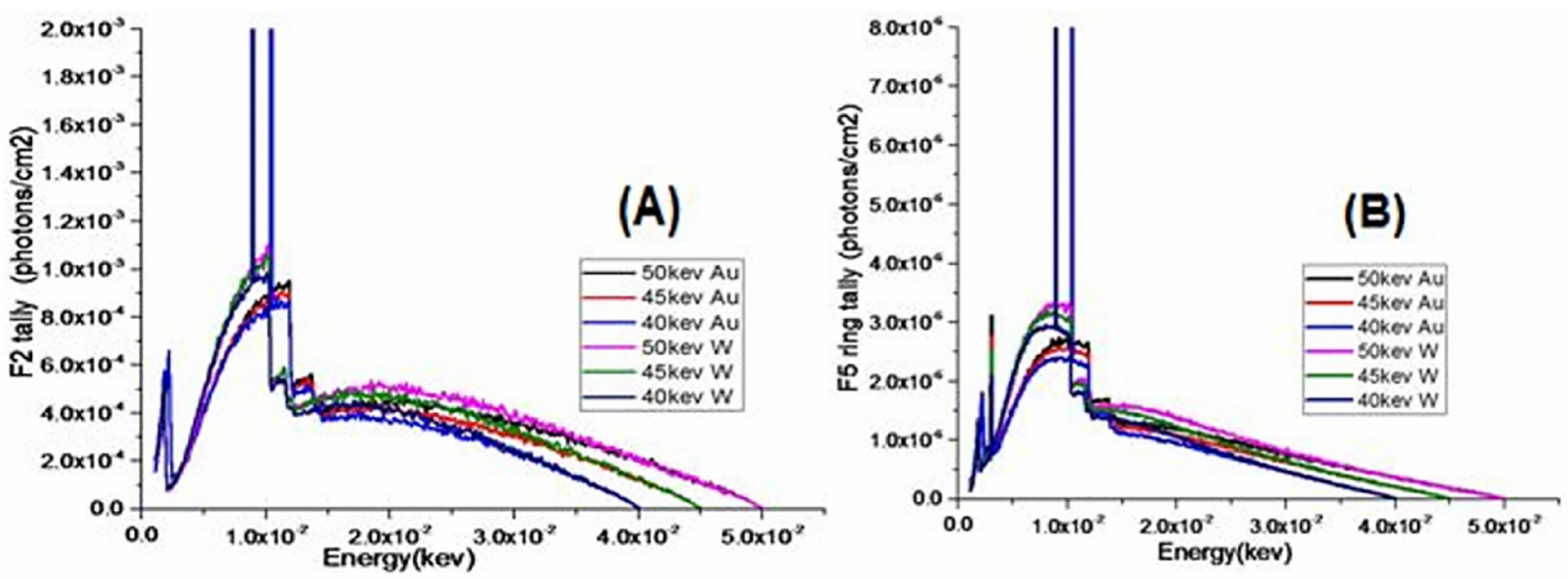

Figure 3. Spectra of X-ray inside the target (A) and outside the target (B)
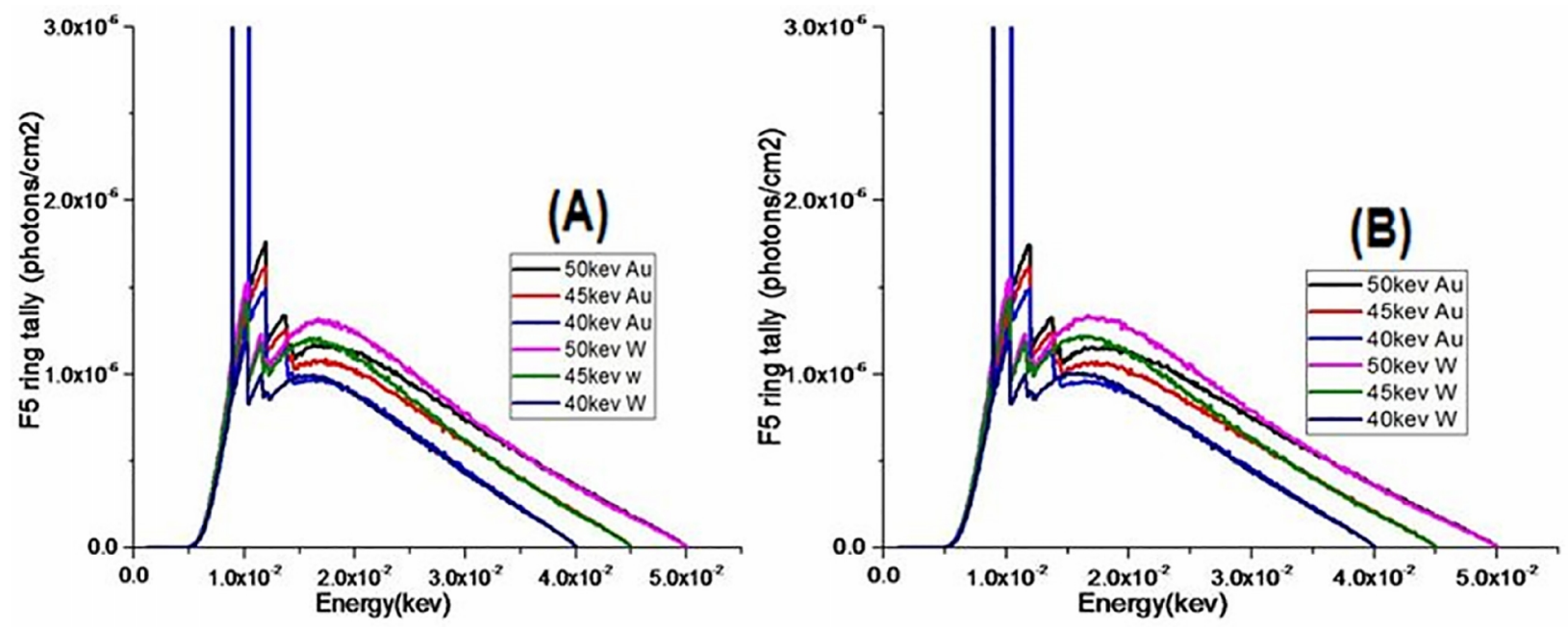

Figure 4. Spectra of $\mathrm{x}$-ray in water (A) and air (B) after the beryllium output window

\subsection{Calculation of absorbed dose}

In this part of the work, tallies to be used along with a mesh tally to calculate the absorbed dose were selected. In MCNP (2.6.0), tallies commonly used for dose calculation are F6, *F4, and *F8. The use of each of these tallies for dose calculation has some advantages and drawbacks. However, considering the negligible range of secondary electrons in solid or liquid media at photon energies lower than $200 \mathrm{keV}$ (38), and the presence of negligible radiative losses (39), and since total kerma has about the same magnitude as the collision kerma (38), the absorbed dose can be approximated by collision kerma (39) and therefore F6 tally was recognized as the best option for this calculation. This tally is known as the track length estimate of energy deposition, or as the heating or energy deposition tally, and is faster than other tallies of dose calculation. Considering the symmetry of source about the zaxis, the dose on this axis was calculated using cylindrical cells. This dose was calculated in the presence of beryllium output window and also with the use of water instead of beryllium. Given the sensitivity of the work, a benchmark with different thicknesses and radii was conducted to assess the setting. In the end, 10 consecutive 
cylindrical cells with identical height and radius were used to calculate the axial dose. The average particle records run with F6 tally to calculate the axial dose in both presence and absence of beryllium and all three energy levels for gold and tungsten targets were, respectively, $163 \times 10^{6}$ and $176 \times 10^{6}$. The radial dose too was calculated with the assumption of symmetry of source around the z-axis. Radial (transverse) dose was calculated using 19 concentric cylindrical cells with identical thickness, but with radii of $0-15 \mathrm{~mm}, 15-30 \mathrm{~mm}$, and so on until the last cell. In this part of work, the radial dose at all energy levels was calculated using average particle records of $301 \times 10^{6}$ and $282 \times 10^{6}$ respectively for the hemispherical-conical and the hemispherical source. Here, the running time of the program tasked with calculating the radial dose of each source was 1000 minutes. It should be mentioned that calculation of axial and radial dose was carried out for a sphere of water with a radius of $20 \mathrm{~cm}$. Also, to prevent overlap between the cells, they were assumed to be a miniscule distance apart. In this study, each 6 programs were run concurrently by a computer with the mentioned specifications, and the average statistical error of these programs along both axes ranged from hundredth of a percent to $2.7 \%$. One of the obviously important features of MCNPX (2.6.0) is the presence of various types of mesh tallies with specific applications. The type-1 mesh tally can be used to calculate and display quantities such as tracks, Flux, Dose, and Pedep (photon energy deposition) in the desired part of the structure. In the mesh tally method, the cards TMESH and ENDMD determine the start and end of the mesh tally frame and the cards CORA, CORB, and CORC determine the mesh size. The mesh can be defined with Cartesian, cylindrical or spherical coordinates based on existing structure. To calculate the dose via a mesh tally method, after preparing the input file, the type-1 mesh tally card was inserted at the end of this input file to calculate the Pedep (equivalent to F6 tally). Considering the symmetry of source about the z-axis, the radial dose was calculated using the cylindrical coordinates in the form of cmesh1: p Pedep, coral $04 \mathrm{i} 3$, corb1 $2.255 \mathrm{i} 2.55$, corc 1 $0.0111 \mathrm{i} 360$ (mesh tally frame is independent of the problem geometry). The execution of MCNPX code (2.6.0) created a binary Mdata file, which was then converted with the gridconv to a format that could be read with Tecplot. Tecplot software was then used to convert the data into a mesh plot with calculated dose values presented over the geometry

\section{Results and discussion}

Figure 5 shows graphs that by using them, the size of the optimum thicknesses of miniature sources was obtained for energies 40,45 , and $50 \mathrm{~kg}$, values of $0.99,1.08$, and $1.34 \mu$ for tungsten target, the values of $0.92,1.01$, and 1.06 respectively for the gold target. Figure 3 shows the spectrums by their use, the production of the photon flux in tungsten and gold targets were compared. Target photon flux generated in tungsten and gold targets were compared. Figure 5 shows the spectrum that by their use, the values of 8.9, 10.2 and $11.6 \mathrm{keV}$ were obtained for energy peaks characteristic for tungsten target, and values of 10.4, 11.8 and $13.8 \mathrm{keV}$ respectively for the gold target. In this study, axial and radial doses were computed for tungsten and gold targets for the applied energies, and comparison between them is according to Figures 6-8.
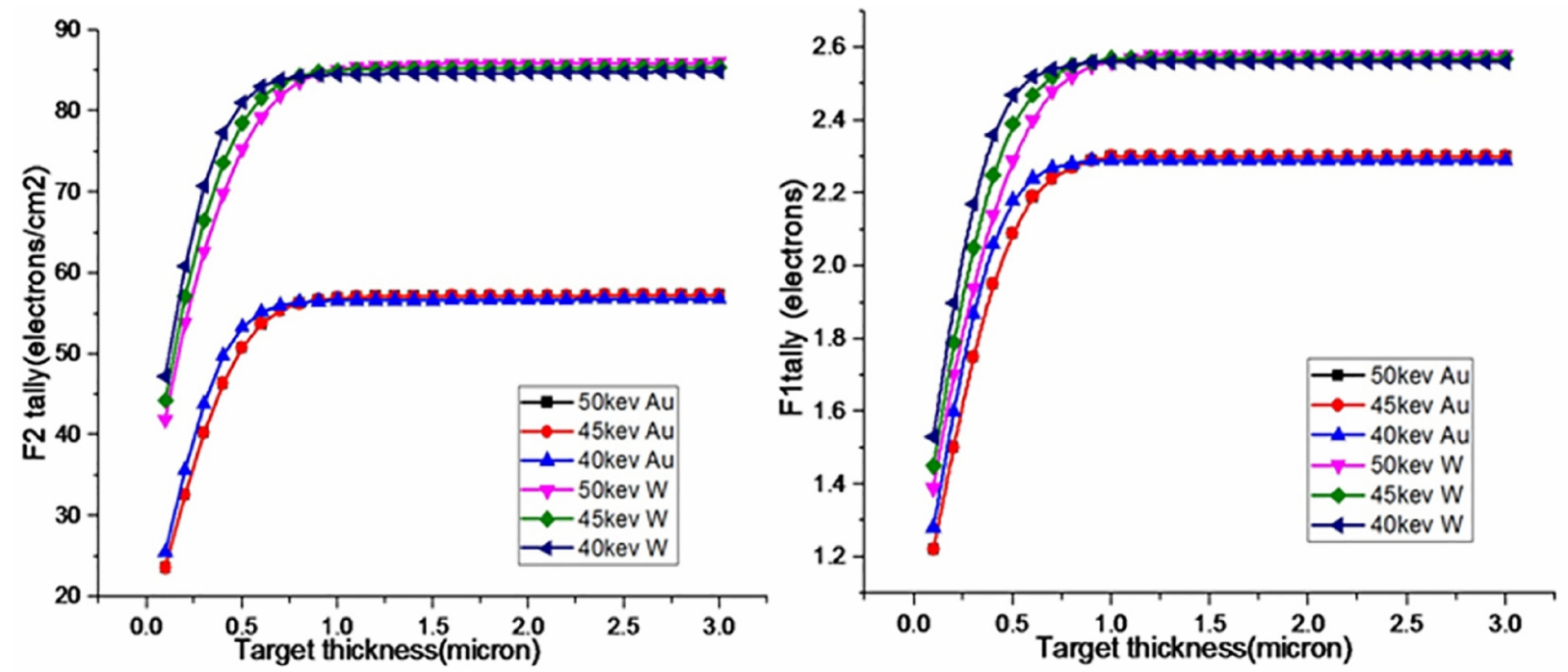

Figure 5. The plots obtained from the tallies used to determine the optimal size of gold and tungsten targets 


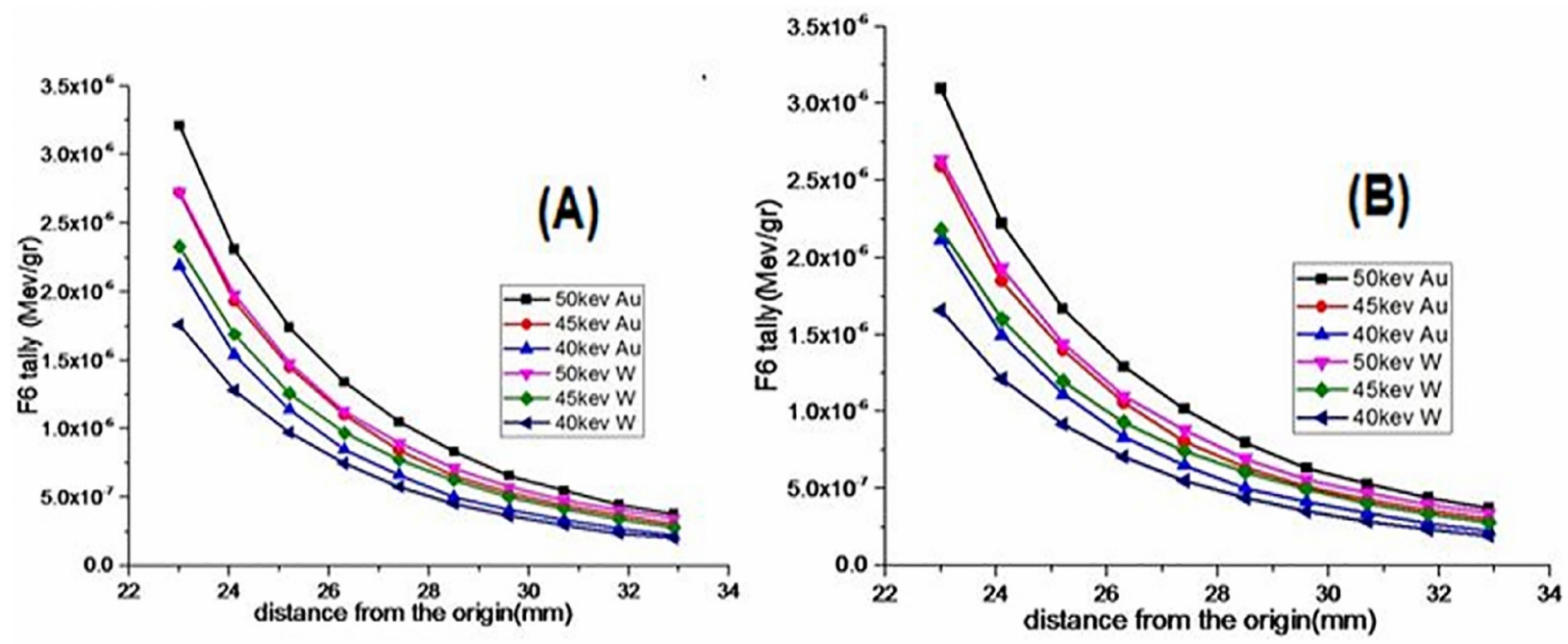

Figure 6. The plots showing the axial dose with beryllium (A) and with water instead of beryllium (B)

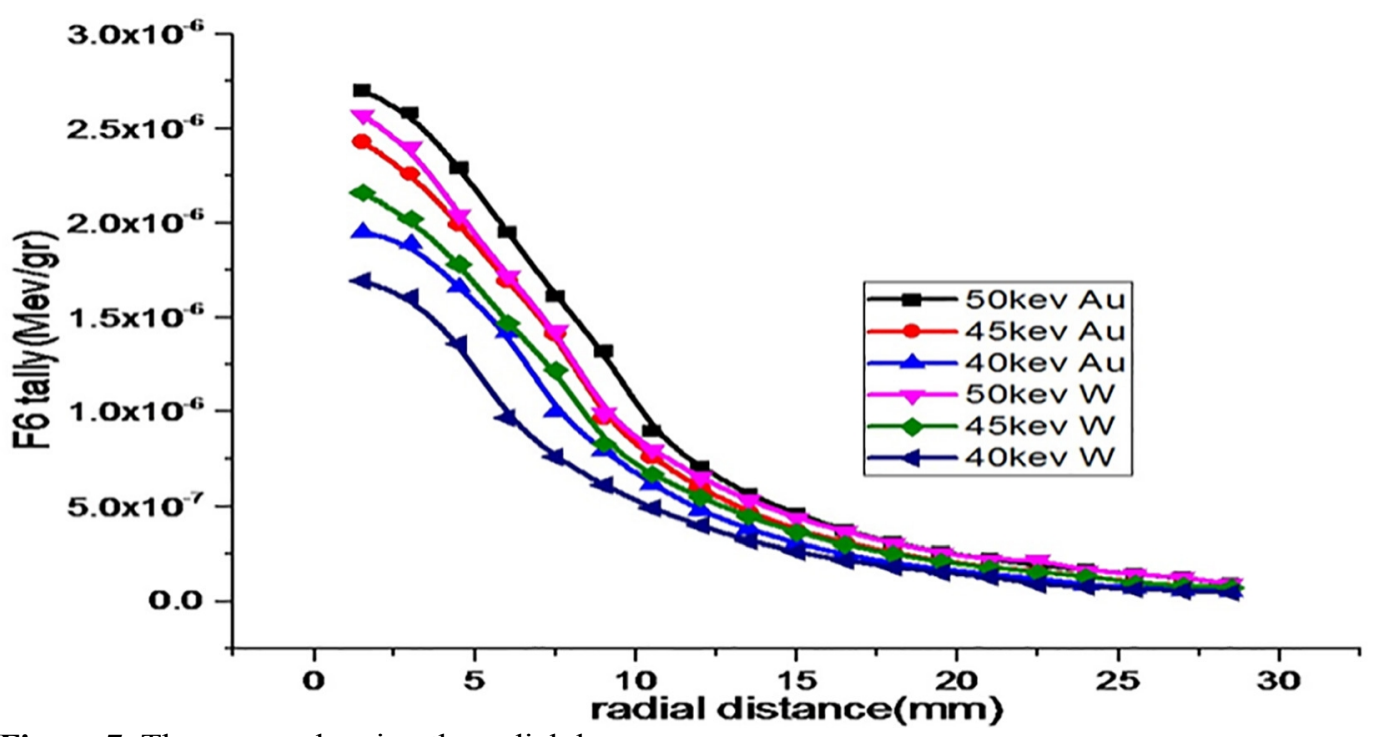

Figure 7. The curves showing the radial dose

\subsection{Evaluation of the factors affecting the optimal target thickness}

In the simulation of this study, two types of thin transmission targets made of tungsten and gold with different atomic numbers, but with the same thickness and energy levels (and almost the same density) are subjected to electron bombardment, so factors determining the produced X-rays (bremsstrahlung and characteristic X-ray) and optimal thickness of the target are the energy of incident electron, and atomic number, thickness and shape of the target. When accelerated, electrons moving with certain energy, collide with tungsten and gold targets, the beam broadening in the target is under heavy influence of the atomic number of target material, and increases with the increase of atomic number (because of more collisions in the target). Also, beam broadening of thinner targets is relatively lower. So overall, the lower atomic number of tungsten, as compared to gold, means that the OT (electron range) of tungsten target rises faster than the gold one. Also note that when the target is thinner than the range of incident electron, some electrons will manage to cross the full thickness of the target and this leads to partial and sub-optimal conversion of electron energy to X-ray (40) (A in Figure 2). So it can be argued that lower thicknesses have a lower attenuation effect and greater thicknesses have a greater attenuation effect on the X-rays produced in the target material. However, in an excessively thick target, photons produced in the first section of the target will be attenuated by the remaining part of the material (41). It can also be argued that using less than the optimum thicknesses decreases the intensity of outgoing photons and average spectrum energy, the use of optimum thickness leads to the maximum intensity of photons, and the use of greater than OTs again decreases the intensity of photons 
and the average spectrum energy, because the target surface may reflect the electrons ( $\mathrm{C}$ in Figure 2). As a result, there must be an optimum thickness (B in Figure 2) based on which most electrons would be converted to photons and the maximum light intensity would be produced. Overall, given that Monte Carlo simulation of both targets was conducted with similar conditions, optimum thickness and X-ray production are affected not only by the energy, target thickness, and atomic number but also by the shape of the target. The plots shown in Figures 3 and 4 illustrate these important effects. According to these figures, for the gold target, the optimum thicknesses of 45 and $50 \mathrm{keV}$ energy levels are relatively close (their corresponding curves are a match and the numerical values are also close), but for the tungsten target, corresponding curves are apart which point to the presence of a different mechanism in tungsten as compared to gold. As the energy level increases, so does the optimal TT, and curve peaks and maxima shift forward, and this demonstrates the effectiveness of all mentioned factors. Figure 9 shows the diagram of the relationship between electron range with the release of energy within the target until it is stopped, so that the optimum thickness is less than the electron ranges and sources with larger optimum thickness, the electron range within them is far greater than the other sources. The line tangent to these curves crosses the x-axis (TT) at the point which represents the range of electrons on that energy level, and this range can be used to determine the OT for the used energy.

\subsection{Exanimation of the obtained spectra and plots}

The curves depicted in Figure 4 show the results of optimization of thickness of tungsten and gold targets with Monte Carlo code MCNPX (2.6.0). These curves show that as target thickness increases, at first, the values of surface flux and flow of electrons at input level increase, but after reaching a maximum value at a certain thickness of surface, flux of electrons at input surface and energy flux and flow of electrons at the output surface remain constant. At output surface, as target thickness increases beyond its optimal value, self-absorption factor increases with thickness and as a result, the surface flux and the surface flow of electrons at the output surface decrease. At the input layer, flow and flux of electrons increase until the same point of OT. All these trends can be clearly seen in the provided plots. The plots also show that, in line with discussions of section 4.1, surface flux and flow production in the tungsten target are far greater than that in the gold target. Figure 5 shows a view of the X-ray spectra produced inside (Figure A) and outside (Figure B) the gold and tungsten targets. At low energy levels, spectra produced inside the gold and tungsten targets had energy peaks of, respectively, $1.7 \mathrm{keV}$ and $2.1 \mathrm{keV}$ and spectra produced outside the gold and tungsten targets had peaks of, respectively, $(3,1.7)$ and $(3,2.1)$. The mean values of the highest energy peak for gold and tungsten targets were $6.12 \times 10^{-3}$ and $5.49 \times 10^{-3}$, respectively. Based on the mean values calculated for energy levels of 40,45 , and $50 \mathrm{keV}$, the energy produced inside the tungsten target is, respectively, $10 \%, 9.7 \%$, and $8.7 \%$ greater than the gold target. The energy flux outside the gold target at energy levels of 40,45 , and $50 \mathrm{keV}$ was estimated to be $11 \%, 10 \%$, and $9 \%$ lower than the tungsten target. The running time of each program was 960 minutes. The statistical error in the calculation of these spectra started at $1 \%$ in the energy level of $1.5 \mathrm{keV}$, reached to a hundredth of a percent in the middle of the spectrum, and increased to $3.5 \%$ in the energy level of $46 \mathrm{keV}$. The errors obtained for energy levels lower than 1.5 or higher than $46 \mathrm{keV}$ were higher than these values. Figure 4 shows the spectra produced in water (A) and air (B) for different levels of the used energy. These spectra show that, the amount of energy obtained for tungsten target water/air ratio $(0.92 \%, 1.4 \%$, and $1.45 \%)$ and gold target air/water $(0.34 \%, 0.31 \%$, and $0.29 \%)$ is more at energy levels of 40,45 , and $50 \mathrm{keV}$, respectively. Furthermore, the spectra depicted in Figure 4 show that the highest energy peak in tungsten and gold targets are, respectively, $6.34 \times 10-6$ and 9.66 $\times 10-6$. The error in calculation of these spectra started at $2 \%$ in the energy level of $4.4 \mathrm{keV}$, reached down to a hundredth of a percent in the middle of the spectrum, and increased to $3 \%$ in the energy level of $48 \mathrm{keV}$; meanwhile, the errors obtained for energy levels lower than 4.4 or higher than $48 \mathrm{keV}$ were higher than these values. In this stage of analysis, the running time of each program was 3,827 minutes. Examination of spectra depicted in Figures 3 and 4 show continuous X-ray spectra resulting from the electron bombardment of the surface of OTs obtained for hemispherical-conical and hemispherical miniature sources. It can also be stated that X-ray spectrum produced inside the target depends on the energy of incident electron; the spectrum of output beam (its intensity) after the target and before the output window depends on the target geometry (shape and thickness); and the spectrum of output beam (its intensity) after the beryllium window depends on thickness, density, and an atomic number of materials of the output window (here beryllium). But the important point regarding the spectrum of the output beam is that, unlike the bremsstrahlung, the beamed characteristic X-rays have discontinuous energies and as Figures 3 and 4 show, the peaks of spectrum imply the transition of electrons from upper shells to the L-shell. 

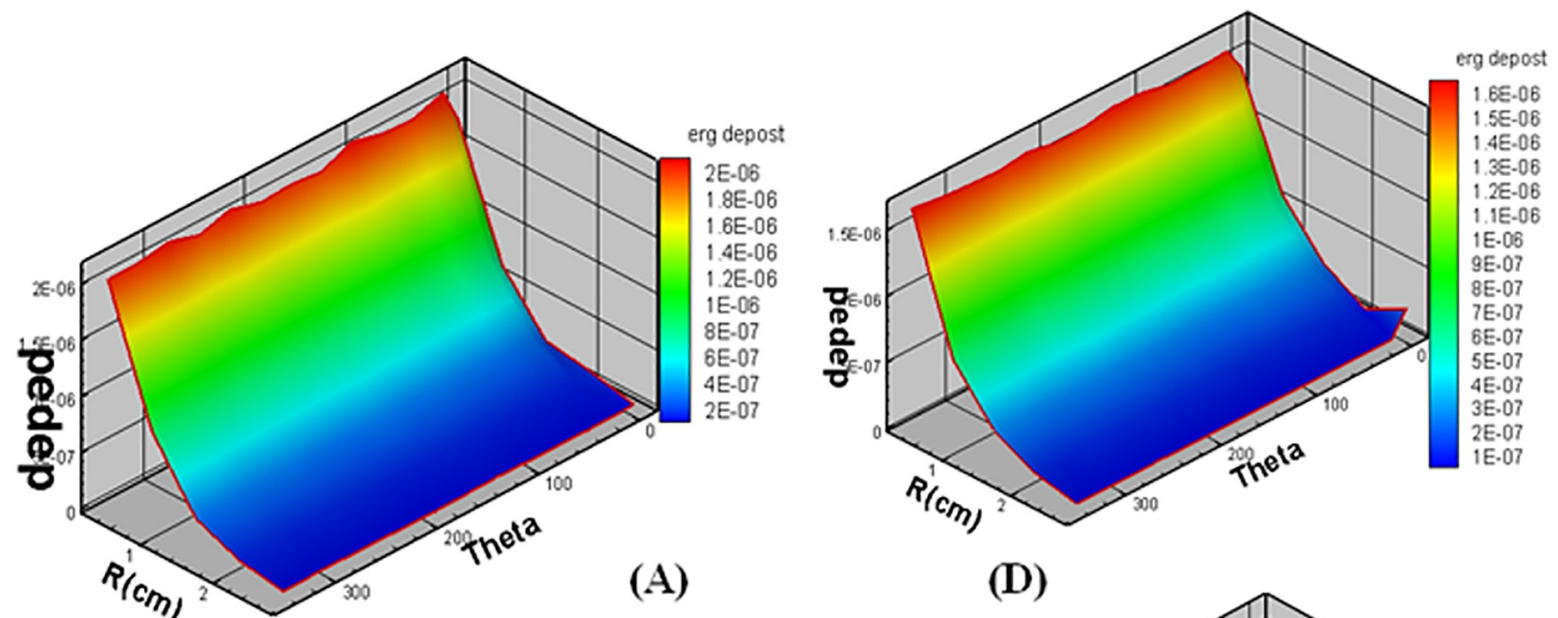

(A)

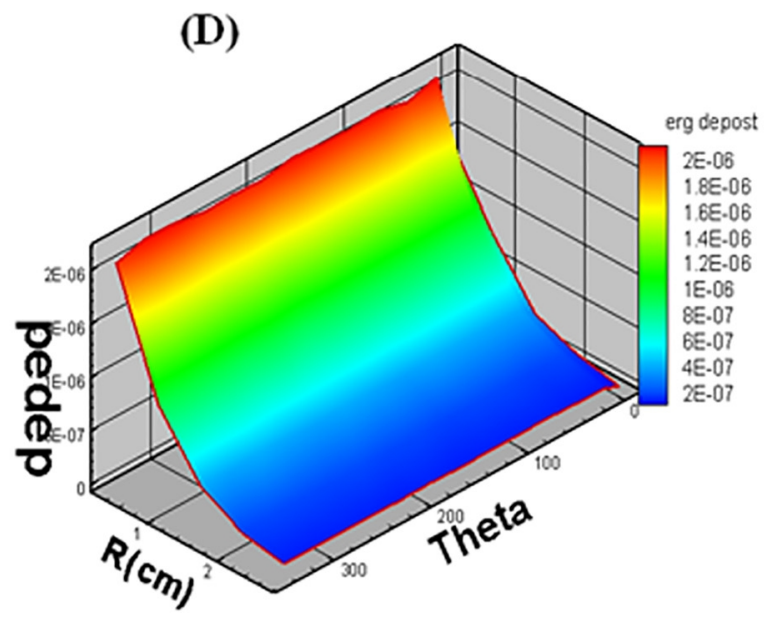

(B)

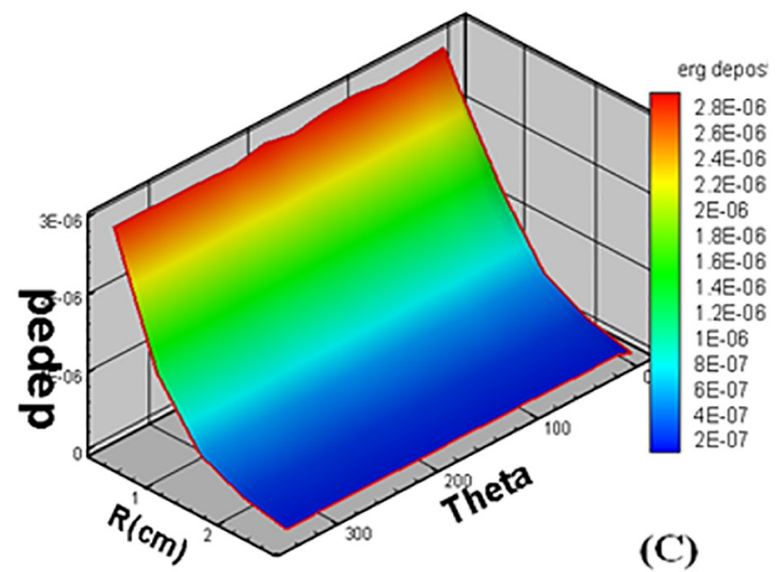

(E)

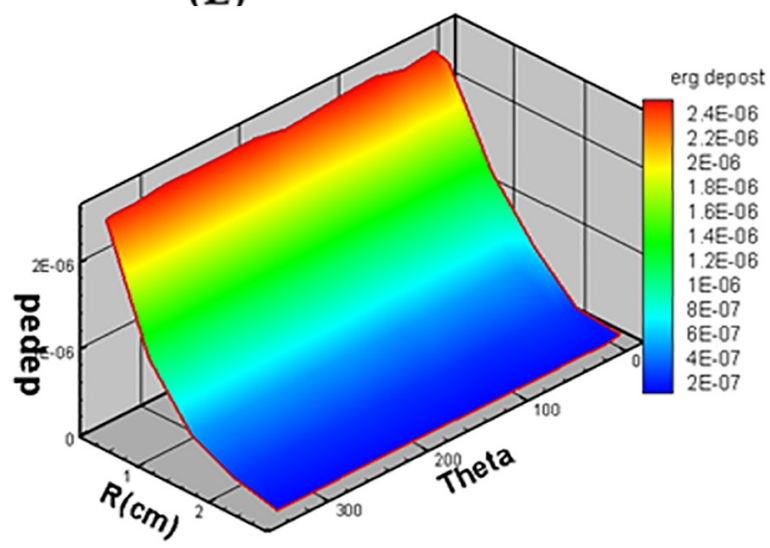

(F)

Figure 8. The plots showing the radial dose with mesh tally: hemispherical (A, B, and C) and hemispherical-conical $(\mathrm{D}, \mathrm{E}$, and $\mathrm{F})$ 

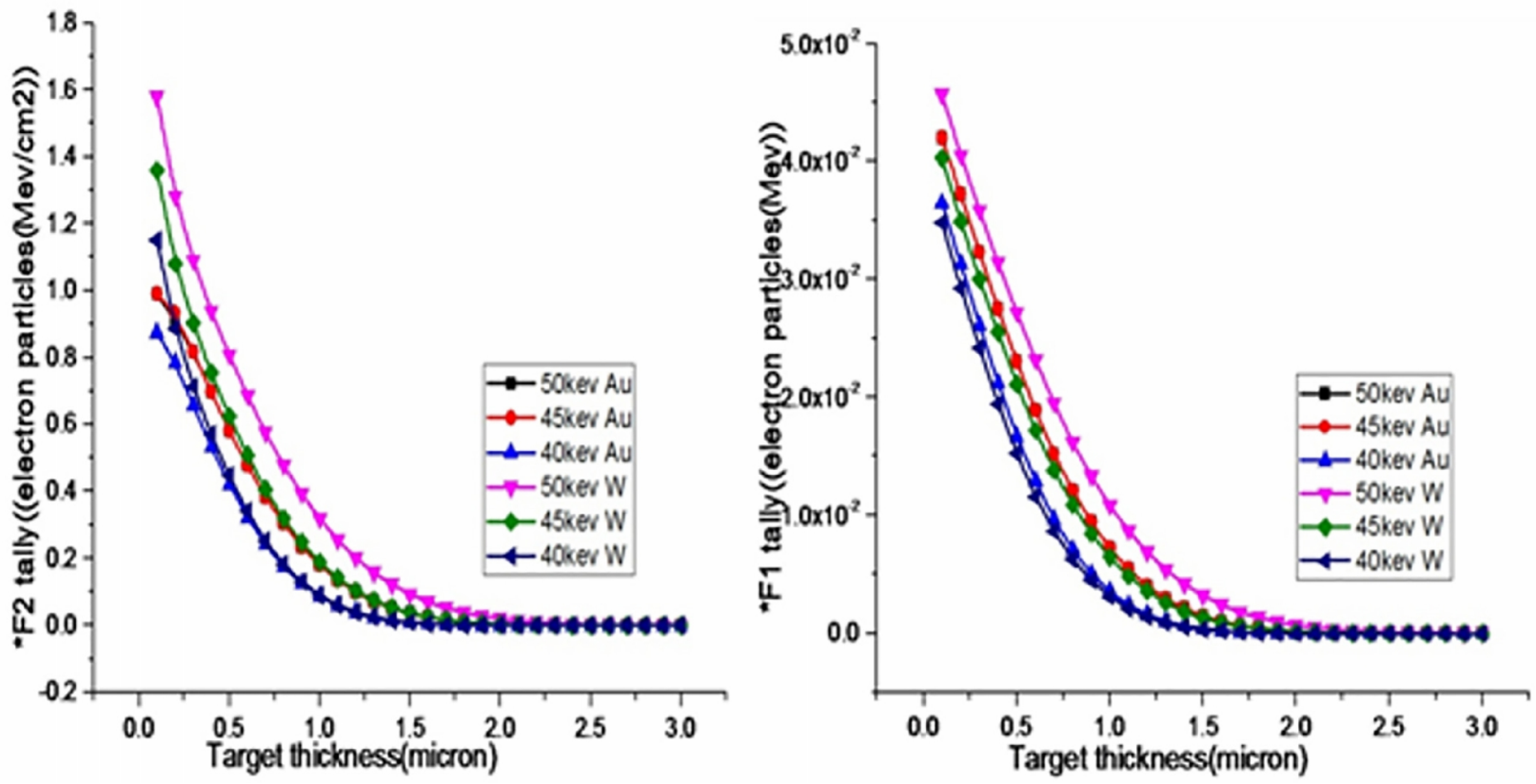

Figure 9. Diagrams obtained from section 2.2 that show electrons range

\subsection{Analysis of the absorbed dose based on the type of tally and mesh tally}

The analysis of dose calculation plots was carried out with two objectives: i) comparing the axial dose in the presence of beryllium as the material of output window with the result obtained with the use of water instead of beryllium, and ii) comparing the radial doses obtained with F6 tally and mesh tally method. Figure 6 shows the plots used for calculation of axial dose with F6 tally. To assess the axial dose, the results of cells with the same number (there were a total of 10 dose calculation cells) but in the models with different energy levels $(40,45,50 \mathrm{keV})$, source shapes (hemispherical and hemispherical-conical) and output window materials (beryllium and water) were compared, and the average differences were determined as a percentage. In the presence of beryllium, the doses of hemispherical source at energy levels of 40,45 , and $50 \mathrm{keV}$ were, respectively, $12.83 \%, 8.94 \%$ and $13.15 \%$ higher than those of the hemispherical-conical source, while in the absence of beryllium, the dose differences were $9.1 \%$, $6.2 \%$, and $8.2 \%$ (in the same order). Figures 7 and 8 show the radial dose calculated with F6 tally and type-1 mesh tally. F6 tally reports the dose of each cell directly under the label of the averaged over a cell kerma (in Mev/gr per photon). Type-1 mesh tally however operates as Pedep (photon energy deposition) equivalent to F6 tally, and can be used to calculate the energy deposition per unit volume (Mev/ $\mathrm{cm} 3$ per particle). The difference of mesh tally with F6 tally is that mesh tally gives the dose by converting the flux, while F6 tally gives the dose directly. Also, mesh tally method requires the density of the medium (here, water) to be applied. At this stage of work, the doses calculated with F6 tally and a mesh tally was compared. The greatest dose calculated with mesh tally at energy level was used as reference, and the radial doses calculated with F6 tally were compared with these reference values. This comparison showed that for the hemispherical-conical source, the doses calculated at energy levels of 40, 45, and 50 $\mathrm{keV}$ using the mesh tally were, respectively, $7.5 \%, 2.5 \%$, and $1.6 \%$ higher than those calculated with F6 tally. For the hemispherical source, the doses obtained at energy levels of 40,45 , and $50 \mathrm{keV}$ with mesh tally were $2.5 \%$, $6.5 \%$, and $3.5 \%$ higher than those obtained with F6 tally. This difference can be attributed to the difference in the density of the medium (water) and difference in the origin of the coordinate system used for mesh tally frame with the one defined for the cells of F6 tally. Despite adjustments, this error proved to be persistent. In any case, the axial and radial doses calculated for hemispherical miniature source, were greater than those calculated for hemisphericalconical source. The axial and radial doses calculated for both sources showed a high gradient near the source and a decrease with distance from the source, at a rate of $1 / \mathrm{r} 3$. Data analysis also showed that the presence of beryllium energy window has a greater impact on the hemispherical source than on the hemispherical-conical source.

\section{Conclusions}

It was found that, before being absorbed, the X-ray resulted from the electron bombardment of target must traverse a path which puts its spectrum under the influence of factors such as the energy of incident electron, cross sectional area of produced bremsstrahlung, target shape, the atomic number of material of target, and density of material constituting the energy window. This study concluded that the presence of beryllium window has a greater impact 
on the hemispherical source than on the hemispherical-conical source, which is reflected in the higher absorbed dose. This could be the reason behind the selection of beryllium for the energy window of INTRABEAM system; and the lower impact of beryllium on tungsten is perhaps the reason why Axxent system uses a combination of beryllium and yttrium

\section{Acknowledgments:}

This paper describes part of the results of research that was performed for the Ph.D. degree in field of medical physics (Thesis no. U-94148). The author would like to thank Dr. Stephen D. Davis (McGill University) for his helpful responses to questions asked about the subject of the study.

\section{Conflict of Interest:}

There is no conflict of interest to be declared.

\section{Authors' contributions:}

All authors contributed to this project and article equally. All authors read and approved the final manuscript.

\section{References:}

1) Herrera R. MCNP5 Monte Carlo based dosimetry for the nucletron iridium-192 high dose-rate brachytherapy source with tissue heterogeneity corrections. Florida Atlantic University. 2012.

2) Liu DMC. Characterization of novel electronic brachytherapy system. McGill University. 2007.

3) Dueitt B. Comparisons of External Beam Radiation Therapy, Brachytherapy, and Combination Therapy in the Treatment of Prostate Cancer.

4) Choe KS, Liauw SL. Radiotherapeutic strategies in the management of low-risk prostate cancer. Scientific World Journal. 2010; 10: 1854-69. doi: 10.1100/tsw.2010.179. PMID: 20852828.

5) Gierga DP, Shefer RE. Characterization of a soft $x$-ray source for intravascular radiation therapy. International Journal of Radiation Oncology* Biology* Physics. 2001; 49(3): 847-56. doi: 10.1016/S03603016(00)01510-8.

6) Kim H, Heoa S, Haa J, Choa S. An Optimization of Super-Miniature X-ray Target. Transactions of the Korean Nuclear Society Spring Meeting, Taebaek, Korea. 2011.

7) Pelowitz DB. MCNPXTM user's manual. Los Alamos National Laboratory, Los Alamos. 2005.

8) Dinsmore M. Miniature x-ray source with flexible probe. Google Patents. 2001.

9) Ihsan A, Heo SH, Kim HJ, Kang CM, Cho SO. An optimal design of X-ray target for uniform X-ray emission from an electronic brachytherapy system. Nuclear Instruments and Methods in Physics Research Section B: Beam Interactions with Materials and Atoms. 2011; 269(10): 1053-7. doi: 10.1016/j.nimb.2011.03.001.

10) Rivard MJ, Davis SD, DeWerd LA, Rusch TW, Axelrod S. Calculated and measured brachytherapy dosimetry parameters in water for the Xoft Axxent X-Ray Source: an electronic brachytherapy source. Med Phys. 2006; 33(11): 4020-32. doi: 10.1118/1.2357021. PMID: 17153382.

11) Eaton D. Electronic brachytherapy--current status and future directions. Br J Radiol. 2015; 88(1049): 20150002. doi: 10.1259/bjr.20150002. PMID: 25748070, PMCID: PMC4628482.

12) Adolfsson E, White S, Landry G, Lund E, Gustafsson H, Verhaegen F, et al. Measurement of absorbed dose to water around an electronic brachytherapy source. Comparison of two dosimetry systems: lithium formate EPR dosimeters and radiochromic EBT2 film. Phys Med Biol. 2015; 60(9): 3869-82. doi: 10.1088/0031-9155/60/9/3869. PMID: 25906141.

13) Hiatt JR, Davis SD, Rivard MJ. A revised dosimetric characterization of the model S700 electronic brachytherapy source containing an anode-centering plastic insert and other components not included in the 2006 model. Medical physics. 2015; 42(6): 2764-76. doi: 10.1118/1.4919280. PMID: 26127029.

14) Lam SC, Xu Y, Ingram G, Chong L. Dosimetric characteristics of INTRABEAM ${ }^{\circledR}$ flat and surface applicators. Translational Cancer Research. 2014; 3(1): 106-11.

15) Rivard MJ, Rusch TW, Axelrod S, editors. Radiological dependence of electronic brachytherapy simulation on input parameters. Medical Physics; 2006: AMER ASSOC PHYSICISTS MEDICINE AMER INST PHYSICS STE 1 NO 1, 2 HUNTINGTON QUADRANGLE, MELVILLE, NY 11747-4502 USA.

16) Rusch T, Axelrod S, Smith P. SU - FF - T - 46: Performance of Xoft Flexi Shield TM Flexible X - Ray Shielding in Laboratory Tests and in a Goat Mammary Model. Medical Physics. 2005; 32(6): 1959. doi: 10.1118/1.1997717.

17) White SA, Landry G, Fonseca GP, Holt R, Rusch T, Beaulieu L, et al. Comparison of TG-43 and TG-186 in breast irradiation using a low energy electronic brachytherapy source. Med Phys. 2014; 41(6): 061701. doi: 10.1118/1.4873319. PMID: 24877796. 
18) Jashni HK, Safigholi H, Meigooni AS. Influences of spherical phantom heterogeneities on dosimetric charactristics of miniature electronic brachytherapy X-ray sources: Monte Carlo study. Applied Radiation and Isotopes. 2014; 95C: 108-13. doi: 10.1016/j.apradiso.2014.10.014. PMID: 25464186.

19) Safigholi H, Faghihi R, Jashni SK, Meigooni AS. Characteristics of miniature electronic brachytherapy $x-$ ray sources based on TG-43U1 formalism using Monte Carlo simulation techniquesa). Med Phys. 2012; 39(4): 1971-9. doi: 10.1118/1.3693046. PMID: 22482618.

20) Muralidhar K, Rout BK, Mallikarjuna A, Poornima A, Murthy PN. Commissioning and quality assurances of the Intrabeam Intra-Operative radiotherapy unit. International Journal of Cancer Therapy and Oncology. 2014; 2(4). doi: 10.14319/ijcto.0204.15.

21) Unsworth MH, Greening JR. Theoretical continuous and L-characteristic X-ray spectra for tungsten target tubes operated at 10 to 50kV. Phys Med Biol. 1970; 15(4): 621-30. doi: 10.1088/0031-9155/15/4/001. PMID: 5488137.

22) Hernandez AM, Boone JM. Unfiltered Monte Carlo-based tungsten anode spectral model from 20 to 640 kV. SPIE Medical Imaging; 2014: International Society for Optics and Photonics. 2014; 9033. doi: 10.1117/12.2042295.

23) Sarrut D, Bardiès M, Boussion N, Freud N, Jan S, Létang JM, et al. A review of the use and potential of the GATE Monte Carlo simulation code for radiation therapy and dosimetry applications. Med phys. 2014; 41(6): 064301. doi: 10.1118/1.4871617. PMID: 24877844.

24) Van der Walt de Kock M. Variance reduction techniques for MCNP applied to PBMR/by Marisa van der Walt de Kock. North-West University. 2009.

25) Redd RA. Radiation dosimetry and medical physics calculations using MCNP 5. Texas A\&M University. 2004.

26) Braga MR, Penna R, Vasconcelos DC, Pereira C, Guerra BT, Silva CJ. Nuclear densimeter of soil simulated in MCNP-4C code. International Nuclear Atlantic Conference.Rio de Janeiro, RJ, Brazil. 2009.

27) McKinney G, Durkee J, Hendricks J, James M, Pelowitz D, Waters L, et al. Review of Monte Carlo allparticle transport codes and overview of recent MCNPX features. PoS. 2006; 88.

28) Larsson E. Realistic tissue dosimetry models using Monte Carlo simulations. Applications for radionuclide therapies: Lund University. 2011; 75.

29) Mcconn RJ, Gesh CJ, Pagh RT, Rucker RA, Williams R. Compendium of material composition data for radiation transport modeling. PNNL-15870 Rev. 2011; 1(4). doi: 10.2172/1023125.

30) Larsson E. Dosimetrical studies on a tissue level using the MCNP4c2 Monte Carlo Simulations. 2004.

31) Nasseri MM. Determination of Tungsten Target Parameters for Transmission X-ray Tube: A Simulation Study Using Geant4. Nuclear Engineering and Technology. 2016; 48(3): 795-8. doi: 10.1016/j.net.2016.01.006.

32) Seibert JA. X-ray imaging physics for nuclear medicine technologists. Part 1: Basic principles of x-ray production. J Nucl Med Technol. 2004; 32(3): 139-47. PMID: 15347692.

33) Mark S, Mordechai S. Applications of Monte Carlo Method in Science and Engineering.

34) Ganguly A. Essential Physics For Radiodology And Imaging. Academic Publishers.

35) Hernandez AM, Boone JM. Tungsten anode spectral model using interpolating cubic splines: Unfiltered $\mathrm{x}-$ ray spectra from $20 \mathrm{kV}$ to $640 \mathrm{kV}$. Med phys. 2014; 41(4): 042101. doi: 10.1118/1.4866216. PMID: 24694149, PMCID: PMC3985923.

36) Wang R, Pei L, Huang Z. Study on Calculation of Detector Flux with Monte Carlo Methods. Journal of Nuclear Science and Technology. 2014; 37(sup1): 436-40. doi: 10.1080/00223131.2000.10874923.

37) Ihsan A, Heo SH, Cho SO. A microfocus X-ray tube based on a microstructured X-ray target. Nuclear Instruments and Methods in Physics Research Section B: Beam Interactions with Materials and Atoms. 2009; 267(21): 3566-73. doi: 10.1016/j.nimb.2009.08.012.

38) Davis SD. Air-kerma strength determination of a miniature x-ray source for brachytherapy applications 2009.

39) Pike TL. A dosimetric characterization of an electronic brachytherapy source in terms of absorbed dose to water. The university of wisconsin-madison. 2012.

40) Heo SH, Ihsan A, Cho SO. Transmission-type microfocus x-ray tube using carbon nanotube field emitters. 2007. doi: 10.1063/1.2735549.

41) Williams T. Axial Energy Distribution in Disc-Shaped Tantalum and Aluminium Bremsstrahlung Conversion Targets. Acta Physica Polonica-Series A General Physics. 2009; 115(6): 1180. doi: 10.12693/APhysPolA.115.1180. 